\title{
Youth Violence and Interventions: Insights from a Complex Agent Network Model
}

\author{
Siew Ann Cheong \\ Division of Physics and Applied Physics \\ School of Physical and Mathematical Sciences \\ Nanyang Technological University \\ 21 Nanyang Link, Singapore 637371 \\ Complexity Institute \\ Nanyang Technological University \\ 18 Nanyang Drive, Singapore 637723 \\ cheongsa@ntu.edu.sg \\ Kaixuan Sun \\ Division of Physics and Applied Physics \\ School of Physical and Mathematical Sciences \\ Nanyang Technological University \\ 21 Nanyang Link, Singapore 637371 \\ P110004@e.ntu.edu.sg \\ Jia Ning Leaw*, Rebecca P. Ang ${ }^{\dagger}$, Vivien S. Huan*, \\ Wei Teng Chan§ and Xiang $\mathrm{Li}^{\mathbb{T}}$ \\ Psychological Studies Academic Group \\ National Institute of Education \\ Nanyang Technological University \\ 1 Nanyang Walk, Singapore 637616 \\ *leaw_ning@hotmail.com \\ ${ }^{\dagger}$ rebecca.ang@nie.edu.sg \\ tvivien.huan@nie.edu.sg \\ \$weiteng.chan@yahoo.com \\ Txiang.li@nie.edu.sg \\ Received 4 October 2016 \\ Accepted 3 January 2017 \\ Published 10 February 2017
}

Youth violence is a growing concern in Singapore. To address this complex social issue, we surveyed the psychology, social science, and criminology literature to identify a total of 11 intrinsic (familial, individual, school) and 2 extrinsic (peer) factors linked to youth violence, and also their interdependencies. We then developed a complex agent network model where each

This is an Open Access article published by World Scientific Publishing Company. It is distributed under the terms of the Creative Commons Attribution 4.0 (CC-BY) License. Further distribution of this work is permitted, provided the original work is properly cited. 
complex agent is represented by a complex factor network of the 13 factors along with youth violence, coupled to each other through the extrinsic factors to form a complex social network. We simulated the model using as initial conditions the results from a large-scale school-based survey of the factors and random social ties. We find factors in each complex agent evolving with time under the influences from other factors, and the social ties between agents evolving with time as a result of behavioral imitation between agents. We ran a sensitivity analysis on the model, to find that the model is most sensitive to the parameters linking (1) non-intact family, (2) delinquency in general, (3) school disengagement, (4) peer delinquency, and (5) friends in gang to gang involvement. We also ran a series of intervention scenario simulations, and our results show that it is critical to intervene early, and successful interventions work by tipping the balance between competing intrinsic and extrinsic factors. Mental health professionals and school counsellors can then apply this unique insight from the model to design more effective interventions.

Keywords: Youth violence; complex agent network model; intervention analysis.

\section{Introduction}

Youth violence is becoming a more serious and widespread problem around the world, ${ }^{1,2}$ and we hear more and more reports of homicides and non-fatal assaults where the perpetrators are youths. ${ }^{3,4}$ In fact, most victims of youth delinquents are themselves adolescents. ${ }^{3}$ According to a world report prepared by Krug et al.,$^{5}$ in 2000 alone there are around 199,000 youth homicides worldwide, meaning nine cases per 100,000 in the population. In the United States, the Office of Juvenile Justice and Delinquency Prevention (OJJDP) in the Department of Justice reported that the prevalence rate of youth gangs has increased significantly since declining from its peak in the early 2000s. In particular, data from the National Gang Center shows that gang involvement increased by $6 \%$, and the number of gangs increased by $28 \%$ from 2002 to $2008 .{ }^{6}$ In other parts of the world, the situation is even more alarming. For every 100,000 people, the youth violence rate is 84.4 in Colombia and $50.2 \mathrm{in} \mathrm{El}$ Salvador. Meanwhile, in the Caribbean, the figure is 41.8 for Puerto Rico. Apart from these generally unsafe places, the figure is around 18.0 in the Russian Federation, and 28.2 in Albania of southeastern Europe. ${ }^{5}$

One would think that youth violence is a problem that does not concern Singapore. After all, the Economist Intelligence Unit ranked Singapore as the second safest city in the world (behind Tokyo) in $2015 .{ }^{7}$ However, according to the annual crime brief released by the Singapore Police Force, ${ }^{8}$ the number of youths arrested increased from 3,031 in 2013 to 3,094 in 2014. In particular, the number of youths arrested for rioting increased from 283 in 2013 to 322 in 2014 . These increases may not seem significant to those trained in the physical sciences, but the Singapore Police Force would need to act to reassure the public, especially after a high-profile case on 30 October 2010, where a 19-year-old male was fatally stabbed by four youths, aged between 18 and 21 . The tertiary student suffered 28 injuries to his head, neck, chest and limbs caused by choppers, knives and a screwdriver, according to a newspaper report. ${ }^{9}$ Following this incident, on 8 November 2010, a group of eight youths armed with parangs and metal rods assaulted another group of 20 youths. Six 
victims, aged between 14 and 20, were treated for injuries, according to records from the Singapore Police Force. ${ }^{10,11}$ More recently, on 1 October 2013, a 19-year-old male was slashed at a shopping mall. His attacker, believed to be in his $20 \mathrm{~s}$, was arrested at the scene. The victim was reportedly only quarreling with the assailant following a month-long dispute, before he was stabbed outside the mall. ${ }^{12,13}$

Clearly, violent youths and their behaviors impose a cost to society, not only in terms of lives lost and maimed, but also in real economic and social terms. Left unchecked, delinquent youths are likely to live an adult life of crime. ${ }^{14-16}$ Policing to a level where crime can be prevented is extremely costly, but prosecuting, incarcerating, and rehabilitating criminals after they ended up on the wrong side of the law is no cheaper. The better alternative, experts argued, is to intervene as early as possible, to prevent juvenile delinquents growing up into adult criminals. ${ }^{5,17-19}$ Specifically, the OJJDP Study Group on Very Young Offenders recommends integrated prevention programs at the school and community levels, combining behavior management, social competence building, channeling of energy into positive recreational activities, and mentoring. ${ }^{20}$ However, interventions are in general multimodal, ${ }^{21-24}$ and their effectiveness are frequently difficult to gauge. ${ }^{25,26}$ It is also time-intensive and labor-intensive to comprehensively evaluate programs and interventions. The complexity of this social problem makes modeling and simulation a valuable approach for testing the efficacy of intervention strategies.

It is only very recently that we find modeling work on juvenile delinquency and juvenile crime in the literature. Understandably, the first few papers took a highly simplified approach to the problem, by treating gang membership and violent dispositions as infections, and building systems dynamics models to describe it. For example, Lee and Do developed a model whereby adolescents at risk are divided into four subpopulations: (1) susceptible $(S),(2)$ gang members $(G),(3)$ delinquent gang members $(D)$, and (4) delinquents who are arrested $(L) \cdot{ }^{27}$ In their model, we find intrinsic transitions that depended only on the prevalence of the source subpopulations, for example, the intrinsic transition from $D$ to $L$ occurs at a rate proportional to $D$. We also find extrinsic transitions that depended on the interactions between the susceptible subpopulations and other subpopulations, for example, the extrinsic transition from $S$ to $G$ occurs at a rate proportional to $S(G+D)$. Similar models were published by Peterson in $2008^{28}$ and Sooknanan et al. in $2012 .^{29}$ Like in all epidemic models, the behavior of such models are determined by the reproductive number $R$ : if $R>1$ the youth delinquency epidemic spreads, else if $R<1$, youth violence dies out. The main drawbacks in these system dynamics models are (1) the homogeneous individuals within each subpopulation, and (2) the model parameters are phenomenological, difficult to unpack into societal, familial, school, and personal factors affecting the psychological states of juvenile delinquents, and therefore the model outcomes cannot directly inform intervention strategies.

More recently, Munyo developed a multi-factor economic dynamics model of juvenile crime, where individuals weigh the expected returns from studying, working, and committing crimes. ${ }^{30}$ Whether they choose to commit crimes depend on the 
likelihood of being arrested, how easy it is to escape from prison, and what is the reward of crime. Crime is therefore the outcome of rational decision making. Munyo identified four major factors that explain the spate of juvenile crime in Uruguay: (1) an anemic recovery of wages relative to total income, (2) the more lenient juvenile crime law, (3) the dramatic increase in the escape rate from juvenile correctional facilities, and (4) the outbreak of a paste cocaine epidemic. Basically, the increase in juvenile crime in Uruguay is blamed on a decrease in the cost associated with criminal activity, and the gains from crime outstripping rewards from legitimate sources. In this model, the subpopulations of agents (Study, Work, Crime + Free, Crime + Escape, Crime + Sentenced) are heterogeneous. There are also internal variables like education level and crime proficiency level that change with time depending on the decision made in the previous time step. However, parameters that determine which utility functions dominate decision making are still phenomenological.

In these modeling papers, the large body of work done on youth violence and juvenile delinquency in the literature has not been exploited. Therefore, in Sec. 2 of this paper, we describe a review of the psychology, social science, and criminology literature to identify familial, individual, school, and peer factors known to be associated with youth violence, the interdependencies between such factors, and complex social interactions between youths at risk. We then explain how the interactions between these factors, and their associations with youth violence can be incorporated into a network model of complex agents, i.e., a complex agent network model, or as a social network of agents whose internal states are described by a complex factor network, i.e., a complex network-of-networks model. In Sec. 3, we explain how we use results from a large-scale school survey as initial conditions for the psychological states of our simulated agents to make our study realistic. The model parameters we initialize randomly for the simulation. Because of the lack of complete incidence data for calibration, we performed a sensitivity analysis of the model instead, followed by an intervention analysis. In Sec. 4, we describe how the sensitivity analysis identified three intrinsic parameters (weighing the couplings between non-intact family, delinquency in general, and school disengagement with gang involvement) and two extrinsic parameters (weighing the couplings between peer delinquency and friends in gang with gang involvement) that the model is most sensitive to. We also describe the outcomes of various interventions targeting school engagement or gang involvement or both, for one individual or more, imposed at different times. Our results suggest a critical need for interventions to be timely. We then conclude in Sec. 5.

\section{Model}

Traditionally, psychologists and criminologists working with case studies have focused on factors influencing violent tendencies in individuals. Because of its social nature, we argue that agent-based models are ideally suited to understanding the 
problems associated with youth violence. ${ }^{31}$ In the most sophisticated agent-based model we can imagine, each young individual would be represented by an artificial intelligence agent. Within a setting that mimics the social environments the youths are in, agents would (1) set behavioral goals, (2) react to events and changes in the environment, and (3) interact with other agents. To build a realistic agent-based model that can do all these, we would need to first know how agents set goals, like showing up at school, meeting up with friends, join a gang, or challenge a rival gang to a fight, etc. We also need to know how they react to events and changes in the environment, like comply with a new rule set by their schools, less pocket money, or having their fathers arrested by the police, etc. Finally, we need to know how they interact with other agents, like responding to a taunt by a fellow gang member, or responding to a taunt by a rival gang member, etc. There are thus too few previous studies on the numerous action items in the three categories as inputs for building such an agent-based model.

Alternatively, we can also build simple toy models, not with the goal of fitting to real-world data and make predictions, but to understand what fundamental features in such models can explain qualitatively what we observe in the real world. Physicists like to take this approach, because toy models are much easier to understand qualitatively and quantitatively. From this perspective, the simplest model of youth violence would be an Ising-like spin model (see Fig. 1(a)), where a normal agent would be represented by a down spin $\left(S_{i}=-1\right)$ while a violent agent would be represented by an up spin $\left(S_{i}=+1\right)$. We can then couple the spins into a regular

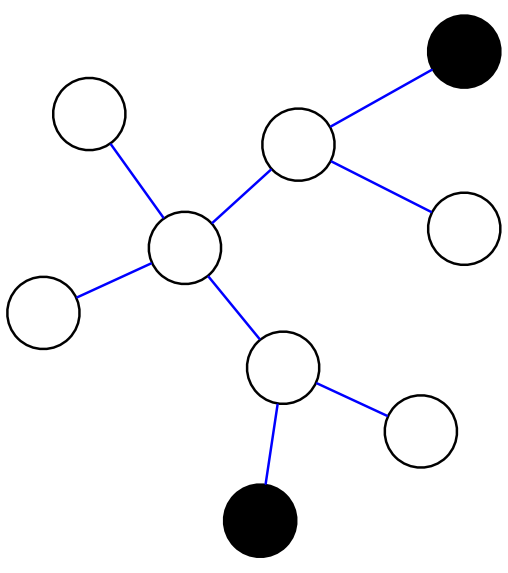

(a)

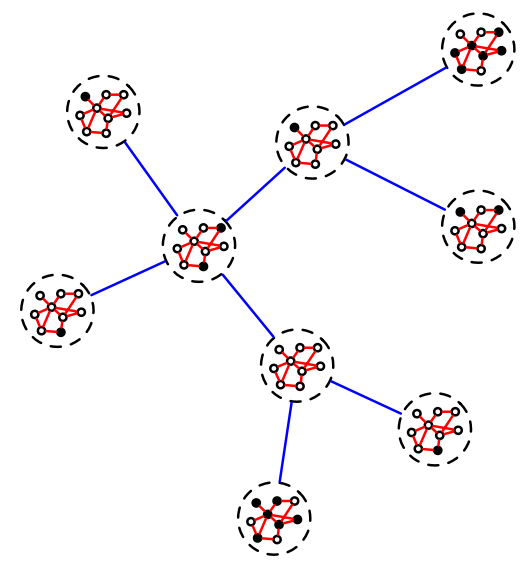

(b)

Fig. 1. (a) A complex network of simple spins. Here, a white circle represents a down spin $\left(S_{i}=-1\right.$, normal agent) while a black circle represents an up spin $\left(S_{i}=+1\right.$, violent agent), and blue links represent interactions between them. (b) A complex network of complex networks. Again, a white circle represents a down spin $\left(S_{i}=-1\right.$, normal factor $)$ and a black circle represents an up spin $\left(S_{i}=+1\right.$, violent factor $)$, but we distinguish between two types of links: red links between factors within an individual agent, and blue links between individual agents. 
lattice, or more 'realistically' into a complex social network. To mimic the small number of violent incidents in real life, and how violence tends to spread, we can make the coupling between spins ferromagnetic, and have the system in a symmetry broken state $(\langle S\rangle \approx-1)$ below the critical temperature, or have the system to be in an external downward field (so that most of the spins are down). Either way we can study how an up spin nucleate a transient up-spin domain, or one that continues growing.

Unfortunately, whether we choose to interpret $S_{i}=+1$ as individual violent incidents, or a violent individual, the Ising-like model described above is unrealistic in the sense that a spin switches quickly from $S_{i}=+1$ to $S_{i}=-1$. In the real world, it is possible to find a normal individual quickly turning violent, but most violent individuals remain violent for a long time unless they undergo counseling or psychiatric therapy. To modify the Ising-like model to make it more realistic, we therefore have to assume that individuals are not simple spins, but have complex internal structures. This can be done by replacing the Ising spin $S_{i}$ with a complex network of spins (see Fig. 1(b)). In this model, we can introduce heterogeneity at two levels. First, red links between factors within an individual agent are strong, while blue links between individual agents are weak. Second, the average strengths of red links can vary from individual to individual. An agent with many violent factors is violent, whereas an agent with few violent factors is normal. More importantly, a violent agent can remain violent even if it is surrounded by normal agents, if its internal links are stronger than its external links. We now understand the cascading phase transitions in such models well because of earlier work on synchronization in modular networks, ${ }^{32-35}$ and more recent work on phase transitions in network of networks. ${ }^{36-39}$

Because we are interested in testing interventions, we impose a deterministic difference equation dynamics on network-of-networks model. In Sec. 2.1, we will describe the factor network model of a complex agent, explaining what each of the factors means, and providing references to studies that showed the interdependencies between factors. We will then describe in Sec. 2.2, how complex agents can be coupled together to form a complex social network of factor networks, where we find links from the gang involvement and delinquency in general factors in one agent to the gang involvement factor in another agent.

\subsection{The factor network model of a complex agent}

Through a review of the psychology, social science, and criminology literature, we find that many factors have been suggested, but only 11 intrinsic factors and 2 extrinsic factors have been linked to youth violence at levels that are statistically significant. These are listed in Table 1 along with their symbols, and will be the variables in our model. For example, $\mathrm{DG}_{i}(t)$ is the level of delinquency in general for agent $i$ at time $t$. In the literature, these factors are measured using a variety of scales. Selecting the appropriate scale for a factor depends on the purpose and design of the study. To keep our simulations simple to interpret, we restrict all variables to 
Table 1. Factors linked to youth violence (VI) in the psychology, social science, and criminology literature and their symbols, organized according to whether they are familial, individual, school, or peer.

\begin{tabular}{clc}
\hline S/No. & \multicolumn{1}{c}{ Factors } & Symbol \\
\hline & Familial & \\
1 & Non-intact family & NF \\
2 & Poverty & PV \\
3 & Father criminality & FC \\
& Individual & \\
4 & Psychopathy & PC \\
5 & Delinquency in general & DG \\
6 & Proactive aggression & PA \\
7 & Gang involvement & GI \\
8 & Substance abuse & SA \\
9 & Age at first offence & AF \\
& School & \\
10 & School disengagement & SD \\
11 & Drop out & DO \\
& Peer & \\
12 & Peer delinquency & PD \\
13 & Friends in gang & FG \\
\hline
\end{tabular}

the unit interval $[0,1]$. We can think of this as normalization of the different scales used for different variables (which is indeed what we did in Sec. 3.1), or as the intensity of each variable over a cross-section of indicators, or as some fuzzy truth level for each variable.

If the factors identified in the literature were independent, we would obtain the model shown in Fig. 2(a). However, we find in the literature that these factors are interdependent. In Table 2, we list these interdependencies, and the references confirming the statistical significance of these connections. Once these interdependencies between factors are included, we obtain the complex factor network shown in Fig. 2(b). Now, because the factors are interdependent, it is natural to wonder whether there are factors that are dominant, because they influence youth violence directly as well as indirectly, and factors that are redundant, because their influence on youth violence can be attributed to the influence on them by other factors. To check this, we perform singular value decomposition on the interdependency matrix $A$, whose matrix elements are $A(\alpha, \beta)=1$ if factor $\alpha$ influences factor $\beta$, and $A(\alpha, \beta)=0$ otherwise. We find 10 nonzero singular values, and as shown in the left panel of Fig. 3, the first singular value is significantly larger than the rest. In the right panel of Fig. 3, we examine the first principal component comprising the most influential factors and also the first principal component comprising the most influenced factors. Judging from their amplitudes, SD, PD, and DG are the most influential factors, followed by SA, PC, PV, PA, NF, FG. FC, AF, DO, VI that are the least influential factors. On the other hand, VI, GI, DG, DO, SA are the most 


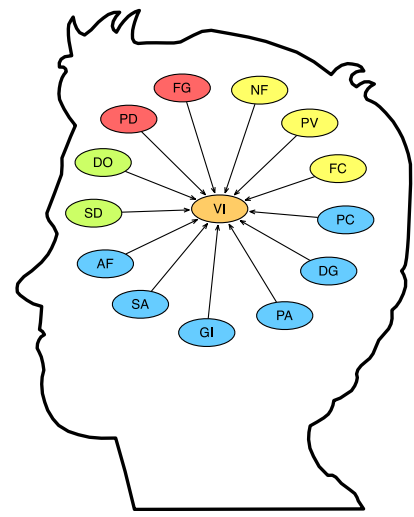

(a)

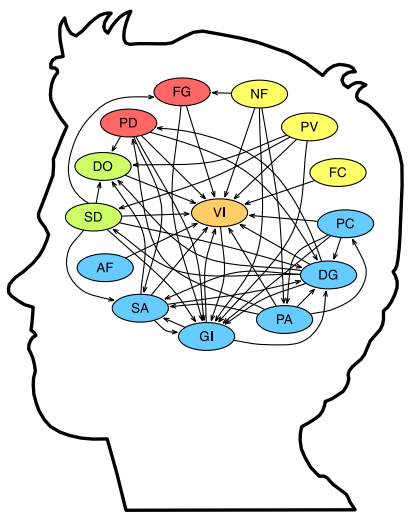

(b)

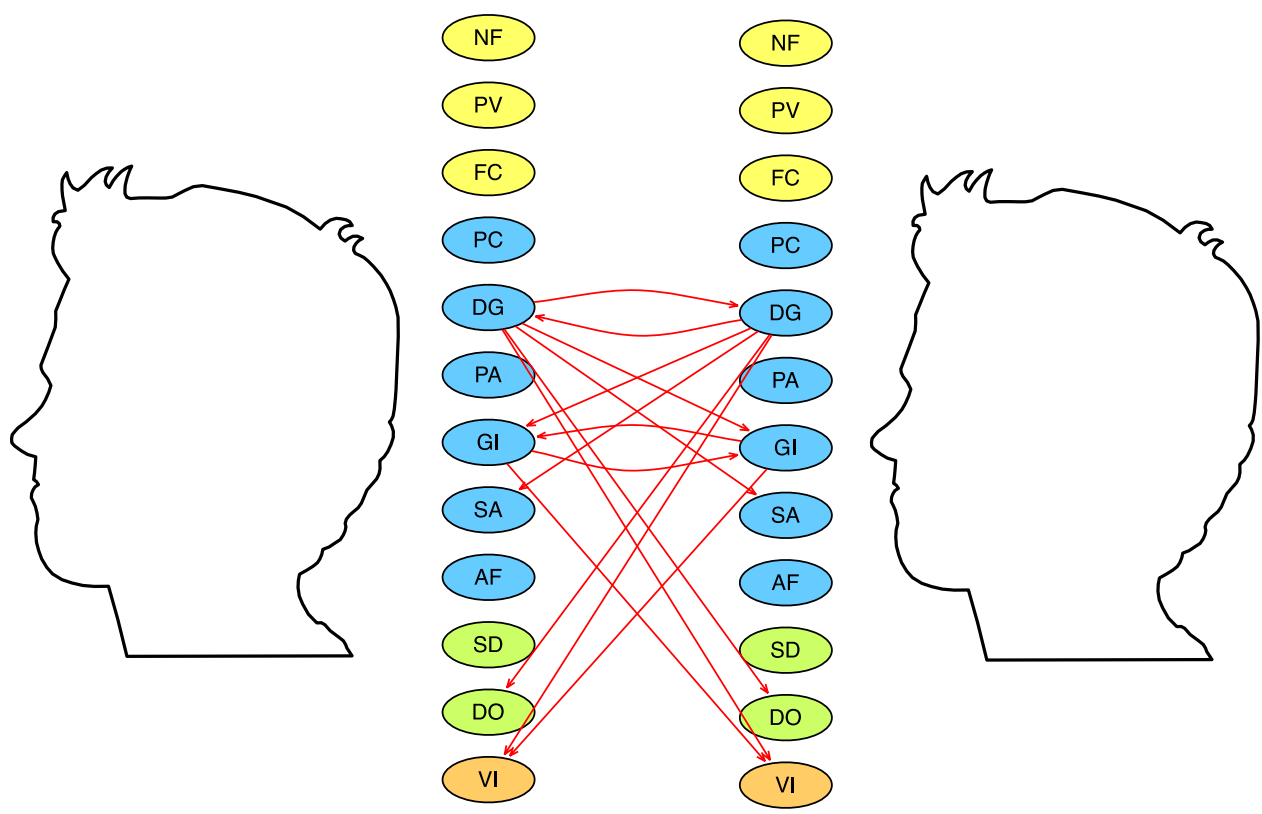

(c)

Fig. 2. (a) Factor model of the 11 intrinsic factors and 2 extrinsic factors linked to youth violence, without interdependencies between factors. (b) Complex network model of the 11 intrinsic factors and 2 extrinsic factors linked to youth violence, including interdependency links between factors. In this figure, familial factors are colored yellow, individual factors are colored blue, school factors are colored green, peer factors are colored red, and youth violence (VI) is colored orange. (c) Unpacking the peer interactions between intrinsic factors of two individuals. In (b), we find arrows from PD to DG, GI, SA, DO, and VI, and an arrow pointing from DG to PD. This means that DG of agent $j$ influences DG, GI, SA, DO, and VI of agent $i$, while DG of agent $i$ influences DG of agent $j$. Similarly, in (b), we find arrows from FG to GI and VI. This means that GI of agent $j$ influences GI and VI of agent $i$. 
Table 2. Interdependencies between factors linked to youth violence. In this table, references are organized into an asymmetric matrix, such that the references in the $\alpha$ th row, $\beta$ th column are for factor $\alpha$ leading to factor $\beta$.

\begin{tabular}{|l|c|c|c|c|c|c|c|c|c|c|c|c|c|c|}
\hline & NF & PV & FC & PC & DG & PA & GI & SA & AF & SD & DO & PD & FG & VI \\
\hline NF & & & & & & {$[40]$} & {$[41]$} & & & & & & {$[42]$} & {$[43,44]$} \\
\hline PV & & & & & & & {$[41]$} & & & {$[45]$} & {$[46]$} & & & {$[44]$} \\
\hline FC & & & & & & & & & & & & & & {$[47]$} \\
\hline PC & & & & & {$[48]$} & {$[49]$} & {$[50]$} & & & & & & & {$[48]$} \\
\hline DG & & & & & & & {$[51]$} & {$[52]$} & & {$[53]$} & {$[54]$} & {$[55]$} & & {$[56]$} \\
\hline PA & & & & {$[49]$} & {$[57]$} & & & {$[58]$} & & & & {$[58]$} & & {$[59]$} \\
\hline GI & & & & & {$[60]$} & & & {$[60]$} & & & & & & {$[43$,} \\
\hline SA & & & & & {$[52]$} & & {$[64]$} & & & & {$[65]$} & & & {$[44]$} \\
\hline AF & & & & & & & & & & & & & & {$[47,61]$} \\
\hline SD & & & & & {$[45,53]$} & & {$[41]$} & {$[45]$} & & & {$[66]$} & & {$[42]$} & {$[51]$} \\
\hline DO & & & & & & & & & & & & & & {$[66]$} \\
\hline PD & & & & & {$[53]$} & & {$[51]$} & {$[58]$} & & & {$[46]$} & & & {$[63]$} \\
\hline FG & & & & & & & {$[64]$} & & & & & & & {$[62]$} \\
\hline VI & & & & & & & {$[41]$} & & & & & & & \\
\hline
\end{tabular}

(a)
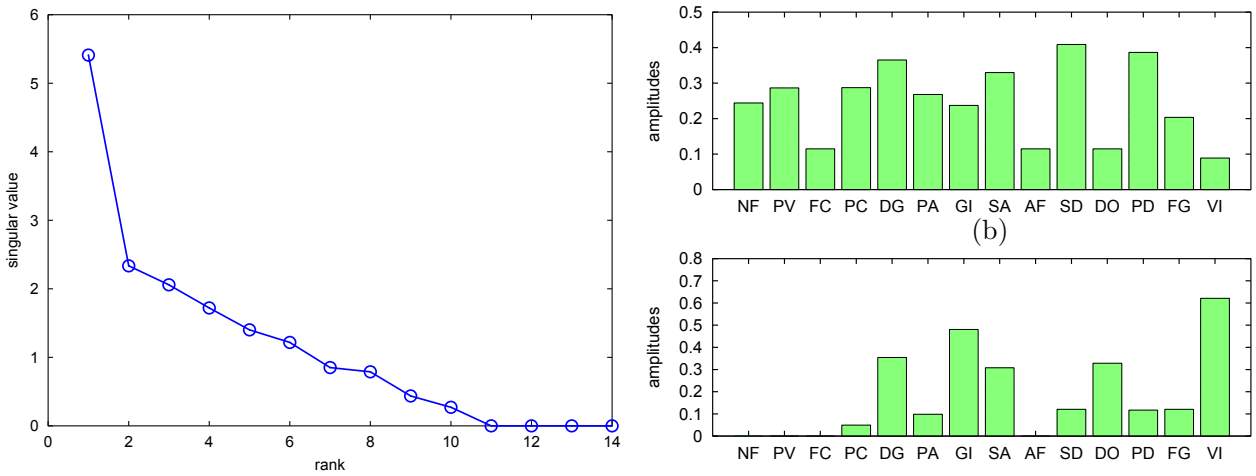

Fig. 3. (left) Singular values of the factor network shown in Fig. 2(b). The first singular value is significantly larger than the other nine nonzero singular values. (right) First-principal-component amplitudes of (a) the most influential factors, and (b) the most influenced factors. 


\section{S. A. Cheong et al.}

influenced factors, followed by PC, PA, SD, PD, FG. NF, PV, FC, AF that are factors that cannot be influenced. Therefore, we learn from the empirical research and theory that we have summarized that while some factors may be more important than others, there is no one dominant factor that can 'explain' youth violence in general. Instead, we need to consider multi-factorial explanations of the kind we demonstrate in this paper.

In this complex factor network, a directed link from factor $\alpha$ to factor $\beta$ means that $\alpha(t)$ contributes to the change $\Delta \beta(t)=\beta(t+1)-\beta(t)$. For example, in Fig. 2(b), we see that there are arrows pointing from PC, PA, GI, SA, SD, and PD to DG. This means that for agent $i, \mathrm{PC}_{i}(t), \mathrm{PA}_{i}(t), \mathrm{GI}_{i}(t), \mathrm{SA}_{i}(t), \mathrm{SD}_{i}(t)$ and $\mathrm{PD}_{i}(t)$ all contribute to $\Delta \mathrm{DG}_{i}(t)$. Unfortunately, previous studies in the literature were not designed to probe the exact functional forms of these dependencies, so we have to make modeling choices along the way. Let us explain how these choices are made, with the intrinsic factor PC as an example. Specifically, we would like the contribution $W_{i}(\mathrm{PC}, \mathrm{DG})$ to $\Delta \mathrm{DG}_{i}(t)$ by $\mathrm{PC}$ to be large when $\mathrm{PC}$ is large, and small when $\mathrm{PC}$ is small. If this is only consideration we have to take care of, then the simplest model we can write down is

$$
W_{i}(\mathrm{PC}, \mathrm{DG}) \propto \mathrm{PC}_{i}(t)
$$

However, we would also like to allow $W_{i}\left(\mathrm{PC}, \mathrm{DG}\right.$ ) to be positive (to increase $\mathrm{DG}_{i}(t)$ ) as well as negative (to decrease $\mathrm{DG}_{i}(t)$ ). This is possible by introducing a reference level for $\mathrm{PC}_{i}(t)$, so that $W_{i}(\mathrm{PC}, \mathrm{DG})$ is proportional to the difference between $\mathrm{PC}_{i}(t)$ and its reference level. Since $\mathrm{PC}_{i}(t)$ is restricted to the interval $[0,1]$, if we set the reference level too close to $0, \mathrm{PC}_{i}(t)$ will make mostly positive contributions. On the other hand, if we set the reference level too close to $1, \mathrm{PC}_{i}(t)$ will make mostly negative contributions. Assuming there is no natural bias towards mostly positive or mostly negative contributions, we set the reference level to $\frac{1}{2}$, so that

$$
W_{i}(\mathrm{PC}, \mathrm{DG}) \propto\left(\mathrm{PC}_{i}(t)-\frac{1}{2}\right) .
$$

Finally, we would like $\mathrm{DG}_{i}(t)$ to be restricted to the interval $[0,1]$ at all times. This means that its rate of increase must slow down when $\mathrm{DG}_{i}(t)$ is close to 1 , and its rate of decrease must slow down when $\mathrm{DG}_{i}(t)$ is close to 0 . For the contribution $W_{i}(\mathrm{PC}, \mathrm{DG})$ by $\mathrm{PV}_{i}(t)$, this can be achieved by setting

$$
W_{i}(\mathrm{PC}, \mathrm{DG})= \begin{cases}w(\mathrm{PC}, \mathrm{DG})\left[\mathrm{PC}_{i}(t)-\frac{1}{2}\right]\left(1-\mathrm{DG}_{i}(t)\right), & \text { if } \mathrm{PC}_{i}(t)>\frac{1}{2} \\ w(\mathrm{PC}, \mathrm{DG})\left[\mathrm{PC}_{i}(t)-\frac{1}{2}\right] \mathrm{DG}_{i}(t), & \text { otherwise }\end{cases}
$$

where $w(\mathrm{PC}, \mathrm{DG})$ is the parameter that determines how strongly $\mathrm{PC}$ influences DG. The overall change to $\mathrm{DG}_{i}(t)$ due to the intrinsic factors PC, PA, GI, SA, and 
$\mathrm{SD}$ is thus

$$
\begin{aligned}
\Delta \mathrm{DG}_{i}(t)= & W_{i}(\mathrm{PC}, \mathrm{DG})+W_{i}(\mathrm{PA}, \mathrm{DG})+W_{i}(\mathrm{GI}, \mathrm{DG}) \\
& +W_{i}(\mathrm{SA}, \mathrm{DG})+W_{i}(\mathrm{SD}, \mathrm{DG})
\end{aligned}
$$

In our model, we assume the factor-to-factor parameters $w(\alpha, \beta)$ are time-independent, and the same for all individuals.

\subsection{The social network model of complex agents}

Now, PD also contributes towards $\Delta \mathrm{DG}_{i}(t)$. Unlike PC, PA, GI, SA, and SD, PD is an extrinsic factor, which means that $\mathrm{PD}_{i}(t)$ is made up of contributions from agents $j$ who are connected to agent $i$. For example, if agent $i$ is connected to agents $j_{1}, j_{2}$, $j_{3}, j_{4}$, and $j_{5}$, then $\mathrm{PD}_{i}(t)$ will receive contributions from $\mathrm{DG}_{j_{1}}(t), \mathrm{DG}_{j_{2}}(t), \mathrm{DG}_{j_{3}}(t)$, $\mathrm{DG}_{j_{4}}(t)$, and $\mathrm{DG}_{j_{5}}(t)$, because these are the delinquency levels of agents $j_{1}, j_{2}, j_{3}, j_{4}$, and $j_{5}$, who are the peers of agent $i$. In Fig. 2(c), we unpack the extrinsic factors PD and FG to show how DG in agent $j$ impacts DG, GI, SA, DO, and VI in agent $i$, and vice versa. Let $0 \leq \sigma_{j i}(t) \leq 1$ denote the strength of the influence agent $j$ has on agent $i$. Then

$$
\mathrm{PD}_{i}(t)=\sum_{j \neq i} \sigma_{j i}(t) \mathrm{DG}_{j}(t), \quad \mathrm{FG}_{i}(t)=\sum_{j \neq i} \sigma_{j i}(t) \mathrm{GI}_{j}(t)
$$

are the sums of weighted contributions of DG and GI respectively from all neighbors $j$ of agent $i$. For $\Delta \mathrm{DG}_{i}(t)$ the contribution by $\mathrm{PD}_{i}(t)$ is thus

$$
W_{i}(\mathrm{PD}, \mathrm{DG})=w(\mathrm{PD}, \mathrm{DG}) \mathrm{PD}_{i}(t)=w(\mathrm{PD}, \mathrm{DG}) \sum_{j \neq i} \sigma_{j i}(t) \mathrm{DG}_{j}(t) .
$$

where $w(\mathrm{PD}, \mathrm{DG})$ is the parameter that determines how strongly $\mathrm{PD}$ influences DG. The overall change to $\mathrm{DG}_{i}(t)$ due to the intrinsic factors PC, PA, GI, SA, SD and the extrinsic factor $\mathrm{PD}_{i}(t)$ is thus

$$
\begin{aligned}
\Delta \mathrm{DG}_{i}(t)= & W_{i}(\mathrm{PC}, \mathrm{DG})+W_{i}(\mathrm{PA}, \mathrm{DG})+W_{i}(\mathrm{GI}, \mathrm{DG}) \\
& +W_{i}(\mathrm{SA}, \mathrm{DG})+W_{i}(\mathrm{SD}, \mathrm{DG})+W_{i}(\mathrm{PD}, \mathrm{DG}) .
\end{aligned}
$$

To complete our model, we need to specify how the social ties $\sigma_{j i}(t)$ are updated. There are very few studies specifying the nature of how social ties evolve between juvenile delinquents. The most relevant publication we can find is Moffitt's dual taxonomy theory, ${ }^{16}$ on which we recently developed a model ${ }^{67}$ In this theory, Moffitt observes that young children are physically and psychologically immature, and have no desire to act like adults. Therefore, they gain no real benefits by going against social norms to do adult things. On the other hand, teenagers are physically and psychologically mature, but society requires them to finish school and get a job before they can assume adult roles. Hence, teenagers will feel that they have gained real benefits by breaking the rules to achieve financial, material, emotional, and sexual independence early through other means. We modeled this benefit as a 


\section{S. A. Cheong et al.}

function that increases linearly with age until $t_{p}$, which is either 17 or 18 years of age depending on the country, when individuals graduate from high school and enter the job market. After $t_{p}$, the benefit becomes constant, because the adult rights are commensurate with adult responsibilities. In the legal systems of developed economies, minors are protected against severe adult punishments when they break the law. Therefore, we model the cost of delinquency as a function that is close to 0 for young individuals, changing sharply at $t_{p}$ to being close to 1 for adult individuals. The other key aspect of Moffitt's theory is how individuals imitate each other's behavior depending on the net benefit (benefit less cost). By simulating this model, we showed the dual taxonomy of life-course-persistent and adolescence-limited delinquents emerges automatically.

In this paper, we also would like to implement this imitation dynamics in the social ties between agents. However, unlike in our recent paper ${ }^{67}$ where the cost and benefit of delinquency change over a period of about 15 years, in this paper our simulations are over two years at most, before the social 'coming of age' $t_{p}$. Hence, we can neglect the cost and benefit, and focus on whether an individual choose to imitate a more delinquent neighbor, or a less delinquent neighbor. It is reasonable to assume that this imitation is facilitated by the peer factors $\mathrm{PD}_{i}(t)$ and $\mathrm{FG}_{i}(t)$. From Fig. 2, we see that PD is influenced by DG and PA. Therefore, we first compute the referenced linear combination

$$
W_{i}(\mathrm{PD})=w(\mathrm{DG}, \mathrm{PD})\left[\mathrm{DG}_{i}(t)-\frac{1}{2}\right]+w(\mathrm{PA}, \mathrm{PD})\left[\mathrm{PA}_{i}(t)-\frac{1}{2}\right],
$$

of agent $i$. If $W_{i}(\mathrm{PD})>0$, agent $i$ is inclined to imitating those more delinquent than itself. Else if $W_{i}(\mathrm{PD})<0$, agent $i$ is inclined to imitating those less delinquent than itself. Consider then the social tie $\sigma_{j i}(t)$, which determines how strongly agent $i$ is influenced by agent $j$. If $W_{i}(\mathrm{PD})>0$ and $\mathrm{DG}_{j}(t)>\mathrm{DG}_{i}(t)$, agent $i$ will imitate the more delinquent agent $j$, and will also increase $\sigma_{j i}(t)$ to do so better. Else if $W_{i}(\mathrm{PD})>0$ and $\mathrm{DG}_{j}(t)<\mathrm{DG}_{i}(t)$, agent $i$ will decrease $\sigma_{j i}(t)$ to prevent being influenced by the less delinquent agent $j$. Conversely, if $W_{i}(\mathrm{PD})<0$ and $\mathrm{DG}_{j}(t)<\mathrm{DG}_{i}(t)$, agent $i$ will increase $\sigma_{j i}(t)$ to better imitate the less delinquent agent $j$. Else if $W_{i}(\mathrm{PD})<0$ and $\mathrm{DG}_{j}(t)>\mathrm{DG}_{i}(t)$, agent $i$ will decrease $\sigma_{j i}(t)$ to prevent being influenced by the more delinquent agent $j$. As we can see, the agency to change the social tie $\sigma_{j i}(t)$ lies with agent $i$ alone, so that

$$
\Delta \sigma_{j i}^{\mathrm{PD}}(t)=\left[\mathrm{DG}_{j}(t)-\mathrm{DG}_{i}(t)\right] W_{i}(\mathrm{PD})\left[1-\sigma_{j i}(t)\right],
$$

if $\left[\mathrm{DG}_{j}(t)-\mathrm{DG}_{i}(t)\right] W_{i}(\mathrm{PD})>0$; and

$$
\Delta \sigma_{j i}^{\mathrm{PD}}(t)=\left[\mathrm{DG}_{j}(t)-\mathrm{DG}_{i}(t)\right] W_{i}(\mathrm{PD}) \sigma_{j i}(t),
$$

if $\left[\mathrm{DG}_{j}(t)-\mathrm{DG}_{i}(t)\right] W_{i}(\mathrm{PD})<0$ facilitates the direction of change desired by agent $i$, and ensures also that $\sigma_{j i}(t)$ remains within the unit interval $[0,1]$. Notice there is no reference level for $\sigma_{j i}(t)$, because the social tie is used to both increase and decrease delinquency in agent $i$. 
Similarly, FG is influenced by NF and SD, so the relevant linear combination is

$$
W_{i}(\mathrm{FG})=w(\mathrm{NF}, \mathrm{FG})\left[\mathrm{NF}_{i}(t)-\frac{1}{2}\right]+w(\mathrm{SD}, \mathrm{FG})\left[\mathrm{SD}_{i}(t)-\frac{1}{2}\right]
$$

for agent $i$, and

$$
\Delta \sigma_{j i}^{\mathrm{FG}}(t)=\left[\mathrm{GI}_{j}(t)-\mathrm{GI}_{i}(t)\right] W_{i}(\mathrm{FG})\left[1-\sigma_{j i}(t)\right],
$$

if $\left[\mathrm{GI}_{j}(t)-\mathrm{GI}_{i}(t)\right] W_{i}(\mathrm{FG})>0$; and

$$
\Delta \sigma_{j i}^{\mathrm{FG}}(t)=\left[\mathrm{GI}_{j}(t)-\mathrm{GI}_{i}(t)\right] W_{i}(\mathrm{FG}) \sigma_{j i}(t),
$$

if $\left[\mathrm{GI}_{j}(t)-\mathrm{GI}_{i}(t)\right] W_{i}(\mathrm{FG})<0$. Combining the two contributions, we thus have

$$
\Delta \sigma_{j i}(t)=w(\mathrm{PD}, \sigma) \Delta \sigma_{j i}^{\mathrm{PD}}(t)+w(\mathrm{FG}, \sigma) \Delta \sigma_{j i}^{\mathrm{FG}}(t)
$$

as the net change in the social tie going from agent $j$ to agent $i$. With this, we have completely specified our complex agent network model of youth violence.

\section{Simulation}

In this section, we describe in Sec. 3.1 how we use the results of a large-scale schoolbased survey as the initial conditions for our simulations. Unfortunately, in this large-scale school-based survey we were not able to perform measurements to estimate the factor-to-factor parameters $w(\alpha, \beta)$ and social ties $\sigma_{j i}(t)$. Therefore, in Sec. 3.2, we explain how these are randomly sampled from the uniform distribution $U(0,1)$. Thereafter, we list in Sec. 3.3 simulation parameters like the time step size and the total simulation time, before going on to explain in Sec. 3.4 a sensitivity analysis done in lieu of calibration against real data, and describe the intervention analysis done in Sec. 3.5.

\subsection{Large-scale school-based survey}

To better understand juvenile delinquency in Singapore, and understand which selfreported factors contributed to this phenomenon, we surveyed 1027 adolescents ( $58.2 \%$ male, $41.8 \%$ female) between 12 to 19 years of age (Grades 7 through 9 ) from three junior high schools in Singapore. ${ }^{68}$ These three schools randomly selected for the study are public schools situated within neighborhood residential estates and these schools are the most common types of schools in Singapore. The mean age of the adolescents was 14.10 years $(\mathrm{SD}=1.15)$. Adolescents' self-reported ethnic identification was as follows: $65.4 \%$ Chinese, $20.8 \%$ Malay, 6.7\% Indian, $1.1 \%$ Eurasian, and $6 \%$ endorsed others (including all ethnic groups not listed). Adolescents' reports of their parents' marital status were as follows: $81 \%$ married, $2.9 \%$ separated, $12.9 \%$ divorced, $1.7 \%$ never married, and $1.5 \%$ widowed.

This was an anonymous survey and therefore the data are non-identifiable. The survey consisted of four self-report scale measures of psychopathy, reactive and 
proactive aggression, school engagement and delinquency. These measures are well established scales with strong psychometric properties. For details please refer to Ref. 64. In addition to scale measures, we also collected demographic data, and asked adolescents about whether they have ever been involved in gang fights, whether they have ever been on probation and whether they have ever belonged to a gang.

Institutional review board approval for this study was obtained from Nanyang Technological University, Singapore. Parental consent and adolescent assent was obtained prior to data collection. Participation was strictly voluntary and at any point in time, adolescents could refuse or discontinue participation with no penalty. Permission was sought and approval obtained from the Ministry of Education, Singapore, and the respective school principals, prior to conducting the research.

In this large-scale school-based survey, all 13 factors were measured. Of these factors, PC, DG, PA, and SD were assessed on multiple items. For example, PC was assessed on 20 items, each rated from 0 to 2 . We convert these to a single 40-point scale for PC by adding up responses to the 20 items. This was also done for DG, PA, and SD, so that they are measured on 18-point, 24-point, and 95-point scales. AF is self-reported from 0 years of age to 19 years of age, the maximum age in the survey. The rest were simply recorded as 0 (absence) or 1 (presence).

To make our simulations realistic, we use the outcomes of this large-scale schoolbased survey as our initial conditions. After eliminating incomplete records, we ended up with the profiles of 1003 individuals. This ensures that we have a population of heterogenous agents, and since our simulation is initialized using real data, there is no need for calibration and validation where this population profile is concerned. However, all factors must first be normalized, so that their values are between 0 and 1 . Since a first offence at a younger age is generally more predictive of adolescent delinquency, AF is normalized such that its value is 0 if the reported age of first offence is 19 , and 1 if the reported age of first offence is 0 . In the survey, school engagement is measured instead of school disengagement. Therefore, we convert a high school engagement score into a low value for SD, and a low school engagement score into a high value for SD.

\subsection{Factor-to-factor parameters and social ties}

In the large-scale school-based survey, social ties $\sigma_{j i}(t)$ can in principle be measured, but they were not. The factor-to-factor parameters $w(\alpha, \beta)$ cannot be measured through self reporting. Therefore, to run the simulations, we sample random social ties $\sigma_{j i}(t)$ from the uniform distribution $U(0,1)$. In this sense, all agents in our simulations are linked, but not equally strongly. More importantly, since $\sigma_{j i} \neq \sigma_{i j}$, we can have very strong influence by agent $i$ on $j$, but only very weak influence the other way round. For the preliminary study reported in this paper, we set $w(\alpha, \beta)=$ $\frac{1}{7}$ for all $\alpha, \beta$. 


\subsection{Simulation parameters}

In our discrete time simulation, one time step $\Delta t=1$ is equivalent to one day, and we simulate the network of complex agents for one year, $T=365$.

To ensure that the factors do not change too quickly, we further divide $\Delta \alpha$ obtained from (1), (2), and (3) by $N=1003$.

\subsection{Sensitivity analysis}

In principle, to calibrate $w(\alpha, \beta)$ and $\sigma_{j i}$, we would need a longitudinal study tracking a cohort over time, and have psychologists decide at each point in time who in the cohort has become violent. We would then simulate our model for the same duration, starting from the same initial conditions, and vary $w(\alpha, \beta)$ and $\sigma_{j i}$ until the simulated life histories agree with the actual life histories as best as they can. As we can imagine, such an undertaking is forbiddingly expensive and intrusive. Therefore, instead of trying to acquire data to calibrate our model, we perform a sensitivity analysis. Basically, if the simulation outcome is insensitive to changes in a parameter, then it is not important to know the precise value of this parameter. On the other hand, if the simulation outcome is sensitive to changes in a parameter, then this sensitivity is important information, even if we ultimately cannot know the parameter value.

Leaving aside the social ties $\sigma_{j i}$, which we will not test, there are altogether 49 factor-to-factor parameters between the 14 variables (11 intrinsic, 2 extrinsic, and VI; see Fig. 2). In our sensitivity analysis, we change the values of the 49 parameters one at a time, keeping the rest unchanged. We do so in four different ways: (1) increase $w(\alpha, \beta)$ by a factor of $2,(2)$ increase $w(\alpha, \beta)$ by a factor of $1.5,(3)$ decrease $w(\alpha, \beta)$ to $\frac{1}{2}$ of its value, and (4) decrease $w(\alpha, \beta)$ to $\frac{1}{3}$ of its value. We then compare the final number of violent agents in these four scenarios with the number of violent agents in the benchmark simulation, where $w(\alpha, \beta)=\frac{1}{7}$ for all $\alpha$ and $\beta$. We distinguish between positive and negative changes to the final number of violent agents when we identify the parameter that our model is most sensitive to.

\subsection{Intervention analysis}

After identifying parameters $w(\alpha, \beta)$ that our model is most sensitive to, we proceeded to test intervention strategies that target these sensitive parameters, against intervention strategies that do not, and ultimately against the no-intervention benchmark. Specifically, we target SD and/or GI, since our sensitivity analysis tells us that $w(\mathrm{SD}, \mathrm{GI})$ is a very important parameter. All in all, we tested six intervention scenarios:

(1) Find all potentially violent agents, but only change $\mathrm{SD}=1$ to $\mathrm{SD}=0.5$ for one of them after one year, and simulate for another year; 
(2) Change $\mathrm{SD}=1$ to $\mathrm{SD}=0.5$ for all potentially violent agents after one year, and simulate for another year;

(3) Change $\mathrm{SD}=1$ to $\mathrm{SD}=0.2$ for all potentially violent agents after one year, and simulate for another year;

(4) Change $\mathrm{SD}=1$ to $\mathrm{SD}=0.2$, and $\mathrm{GI}=1$ to $\mathrm{GI}=0$ for all potentially violent agents after one year, and simulate for another year;

(5) Change SD from their initial values to 0.5 for all potentially violent agents at the start of the simulation ( $\mathrm{GI}=0$ already), and simulate for one year;

(6) Change all agents with $\mathrm{SD}>0.5$ to $\mathrm{SD}=0.5$, at the start of the simulation, and simulate for one year.

In these scenarios, we varied the extent, forcefulness, and also timing of the intervention.

\section{Results and Discussion}

In this section, we report in Sec. 4.1 how the factors evolve with time. Then in Sec. 4.2 , we report the results of our sensitivity analysis. Finally, we report in Sec. 4.3, the results of our intervention analysis.

\subsection{Time dependence of factors}

From our benchmark simulations, we find that there are factors that evolve quickly (Figs. 4(a)-4(c)), and factors that evolve slowly (Figs. 4(d)-4(f)). As we can see, in some factors (including DG), the factor value decreases rapidly to zero for most agents, and increases rapidly to one for a few agents. In other factors (including SD), the factor value increases and decreases more slowly. But while the factor value decreases to zero or increases to one for most agents, for some agents the factor value tends towards some non-zero constant value over the duration of the simulation. Finally, we find in a number of factors (including VI), the factor value first increases and then decreases, or first decreases and then increases.

\subsection{Sensitivity analysis}

From Fig. 4, we see that after simulating the model for one year, there are five agents with VI = 1 (out of 1003 agents). This number of violent agents can vary a lot, depending on our choice of the reference level in (1). Indeed, this suggests further surveys to measure correlations between the factor values and whom adolescents deem worthy of imitation. In this context, our arbitrary choice of $\frac{1}{2}$ as our reference level can be seen as 'neutral', in the sense in our model agents are not biased in choosing to imitate those more violent than themselves, or less violent than themselves. Therefore, in our sensitivity analysis, we restrict ourselves to measuring the change in the number of violent agents when each of the parameters is changed. These changes are shown as color maps in Fig. 5. 


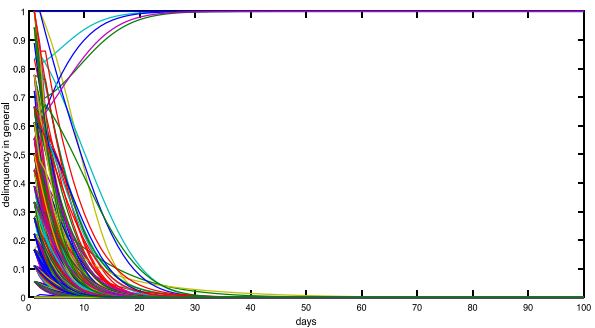

(a)

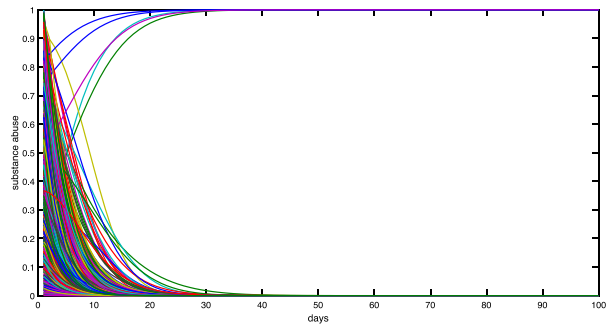

(b)

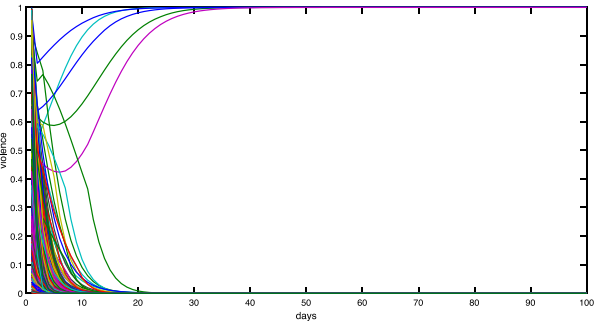

(c)

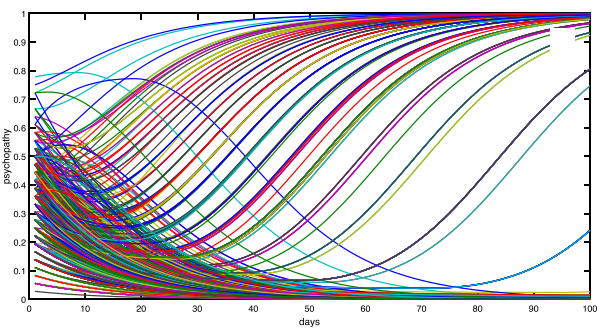

(d)

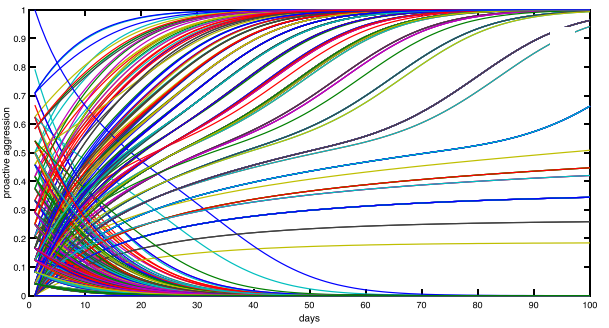

(e)

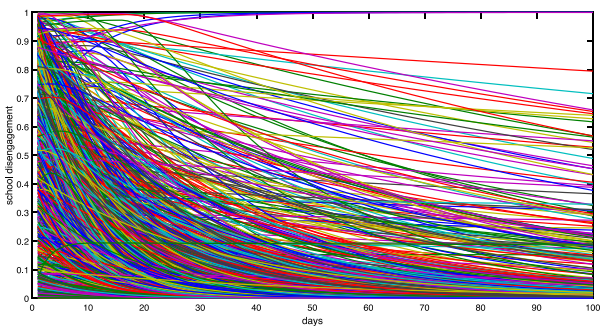

(f)

Fig. 4. The fast time evolution of (a) DG, (b) SA, (c) VI, and slow time evolution of (d) PC, (e) PA, (f) SD for $N=1003$ agents in our model.

We realized that the parameters that produce the largest changes in the number of violent agents when their values are increased $\left(w(\alpha, \beta) \rightarrow \frac{3}{2} w(\alpha, \beta)\right.$ and $w(\alpha, \beta) \rightarrow$ $2 w(\alpha \beta))$ are not the same parameters that produce the largest changes in the number of violent agents when their values are decreased $\left(w(\alpha, \beta) \rightarrow \frac{1}{2} w(\alpha \beta)\right.$ and $\left.w(\alpha, \beta) \rightarrow \frac{1}{3} w(\alpha \beta)\right)$. Therefore, we identify one set of sensitive parameters $(w(\mathrm{DG}, \mathrm{GI}), w(\mathrm{NF}, \mathrm{GI})$, and $w(\mathrm{SD}, \mathrm{GI}))$ for increasing parameter value, and another set of sensitive parameters $(w(\mathrm{PD}, \mathrm{GI})$ and $w(\mathrm{FG}, \mathrm{GI}))$ for decreasing parameter value (see Fig. 5).

By running this sensitivity analysis, we have effectively carried out a review and analysis of the psychology, social science, and criminology literature relevant to this topic area. Naturally, our present scope is limited to $w(\alpha, \beta) \approx \frac{1}{7}$ for all parameters, and we intend to perform a more comprehensive survey of the parameter space in 

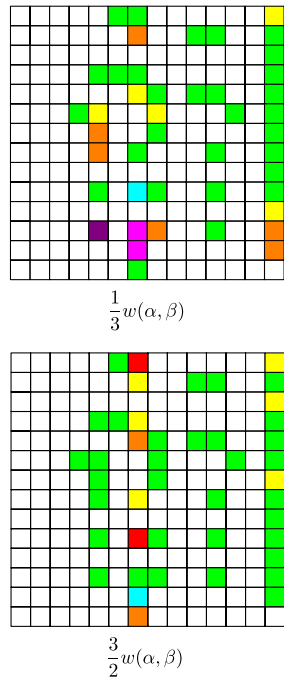
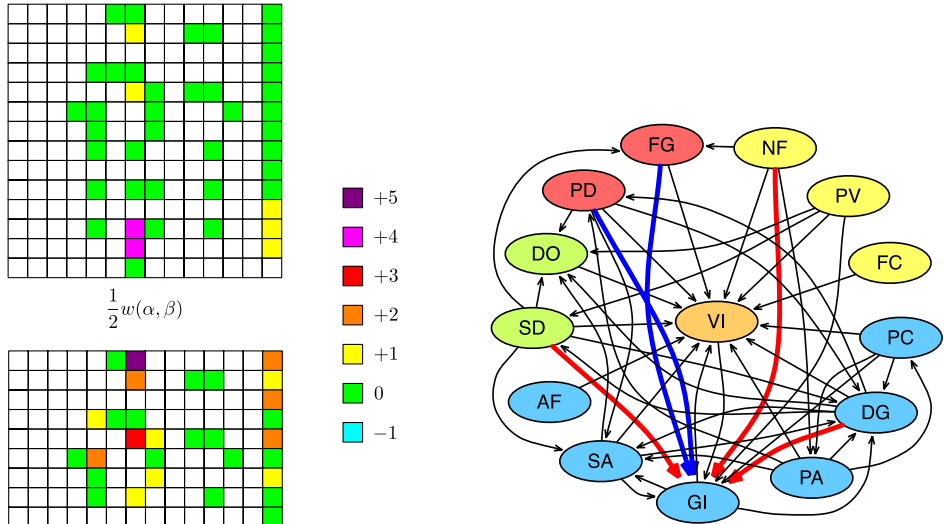

Fig. 5. (left) Color maps of the changes in the number of violent agents when the parameters $w(\alpha, \beta)$ are changed to $1 / 3,1 / 2,3 / 2$, and 2 times their original values. In this figure, the $14 \times 14$ color maps are organized in the same way, the factor-to-factor matrix is in Table 2. (right) The three sensitive parameters for increasing parameter values (red) and two sensitive parameters for decreasing parameter values (blue).

future. Nevertheless, within the narrow scope at present we observed that the simulation outcome (number of violent agents) is sensitive only to a small number of parameters. In future, we also intend to test how sensitive the simulation outcome is to unexplored links between the factors. For example, in the literature we did not find any evidence suggesting that a non-intact family (NF) may directly affect the school disengagement (SD). We could add this link to the model, and simulate it with varying values for $w(\mathrm{NF}, \mathrm{SD})$ to see how strongly it affects the simulation outcome.

More interestingly, we find from our simulations that the number of violent agents increased when $w(\mathrm{PD}, \mathrm{GI})$ and $w(\mathrm{FG}, \mathrm{GI})$ were decreased. This result runs counter to our intuition that PD and FG should be positively correlated with GI. Scrutinizing our simulation results, we found that the five agents that were violent in the benchmark simulation continued to be violent after $w(\mathrm{PD}, \mathrm{GI})$ and $w(\mathrm{FG}, \mathrm{GI})$ were decreased. We therefore focused on the four agents that became violent only after $w(\mathrm{PD}, \mathrm{GI})$ and $w(\mathrm{FG}, \mathrm{GI})$ were decreased. Examining how their VI values evolve over time, and also the DG and GI values of their neighbors, we realized that the simulation outcome for these four agents is decided by the competition between intrinsic and extrinsic factors. More precisely, these four agents are surrounded by 'good' neighbors. Therefore, when $w(\mathrm{PD}, \mathrm{GI})=w(\mathrm{FG}, \mathrm{GI})=\frac{1}{7}$, these 'good' connections drive the VI values of these four agents to zero. When $w(\mathrm{PD}, \mathrm{GI})$ and $w(\mathrm{FG}, \mathrm{GI})$ are decreased, the influences from their 'good' neighbors are diminished, and their own 'bad' intrinsic factors drive their VI values to ultimately saturate. 


\subsection{Intervention analysis}

In scenarios (1) to (4), we first ran a year-long simulation for the five agents to become fully violent, before intervening. It is therefore not surprising that these interventions were all ineffective (no change in the final number of violent agents). In scenarios (5) and (6), we intervened right at the start, and managed to reduce the eventual number of violent agents from 5 to 3 . Scenarios (1) to (5) are targeted scenarios, i.e., we need to know beforehand which agents will eventually become violent, and apply our intervention on them directly. We might object to such a requirement as being unrealistic, but in the untargeted scenario (6), we apply our intervention on all agents with $\mathrm{SD}>0.5$ to change $\mathrm{SD}$ to $\mathrm{SD}=0.5$. We get the same outcome, but would have to put in more resources to target more agents. This tells us that intervention must be timely to be effective.

Finally, instead of intervening right at the start of the simulation, we tested how late into the simulation we can intervene with strategy (6), and still obtain acceptable results. We tried to do so at $t=15,30,60,90$ days of the simulation, and found that only the intervention applied at $t=15$ days was successful. From Fig. 4, we understood why this is so: after about 20 days VI would have reached VI $=0$ for most agents, and VI $=1$ for the violent agents. Beyond this point, none of our intervention strategies work. Although we have taken care with the inputs to make our simulations realistic, we have also made a number of ad hoc modeling choices. Therefore, some outcomes in our simulations will not be realistic. For example, the time scale for the evolution of VI is artificially short because of our choice for $w(\alpha, \beta)$ and $\epsilon$. Certainly, we do not believe that individuals in the real world can go from mildly violent to very violent in a matter of 20 days, leaving us with an impossibly short period of 15 days to intervene. We can easily re-run our simulations with smaller $w(\alpha, \beta)$ and $\epsilon$, so that the average time for VI to saturate matches the average time it takes in the real world for an individual to become violent. Other simulation outcomes will remain qualitatively the same over a broad range of parameters. These are much more likely to be also true in the real world, if we have got the model mostly right. For example, however long it takes for our simulated agents to eventually become violent, there is a finite time window within which we must act to prevent this. We learn therefore from our intervention simulations that early intervention works best, a conclusion that is supported by a large body of empirical research.

\section{Conclusions}

In summary, to better understand the social phenomenon of youth violence and assess the effectiveness of different intervention measures, we developed in this paper a complex agent network model that synthesize 13 statistically significant factors linked to youth violence (Table 1), and their interdependencies (Table 2). 11 of these 13 factors are intrinsic (acting within an individual), while 2 of the 13 factors are 
extrinsic (acting between individuals). The model confirms empirical research and theory in psychology and criminology suggesting that no single factor is capable of explaining youth violence, and that a multi-factorial approach is necessary to gain deeper insights. We simulated how these factors evolve with time under each other's influences, with random initial social ties for the agents, and using the results of a large-scale school-based survey as initial conditions for the factors. We ran a sensitivity analysis of the model, to find it most sensitive to increases in the parameters coupling non-intact family and gang involvement (NF-GI), delinquency in general and gang involvement (DG-GI), and school disengagement and gang involvement (SD-GI), and decreases in the parameters coupling peer delinquency and gang involvement (PD-GI) and friends in gang and gang involvement (FG-GI). We further simulated five targeted intervention strategies and 1 untargeted intervention strategy, with various starting times, to find that for interventions to be effective, they must be imposed shortly after the start of the simulation. Again, this is consistent with the large body of empirical research on interventions, giving us confidence that the model can be useful for the analyses of other intervention and prevention measures. Our model can also guide future studies to discover factors (like a variety of positive influences that can reduce violent tendencies in youths) that can potentially influence youth violence, but have not been systematically tested. The model can also be modified to test deterrent measures like visiting juvenile detention centers and youth gang members who land themselves in hospitals, or the effects of counselling and other interventions to help delinquent youths foresee the future impacts of their present actions.

\section{Acknowledgments}

This research was supported by the MOE AcRF Tier 2 grant (MOE2012-T2-1-079) awarded to Principal Investigator, Rebecca P. Ang, and Co-Principal Investigators Siew Ann Cheong and Vivien S. Huan. We also thank the reviewers for helpful comments to improve the paper.

\section{References}

1. J. S. Hoffman, L. M. Knox and R. Cohen, Beyond Suppression: Global Perspectives on Youth Violence (ABC-CLIO, 2011).

2. Youth Violence: A Report of the Surgeon General (Office of the Surgeon General (US), 2001).

3. A. Reza, E. G. Krug and J. A. Mercy, Inj. Prev. 7 (2001) 104-111.

4. Ad Hoc Committee on Health Research Relating to Future Intervention Options, Investing in Health Research and Development (World Health Organization, 1996).

5. E. G. Krug, J. A. Mercy, L. L. Dahlberg and A. B. Zwi, Lancet 360(9339) (2002) 1083-1088.

6. A. Egley Jr., J. C. Howell and J. P. Moore, Highlights of the 2008 National Youth Gang Survey, OJJDP Fact Sheet (U.S. Department of Justice, 2010). 
7. The Safe Cities Index 2015: Assessing Urban Security in the Digital Age (The Economist Intelligence Unit, 2015), Available at http://safecities.economist.com/wp-content/ uploads/2015/01/EIU_Safe_Cities_Index_2015_white_paper-1.pdf.

8. Annual Crime Brief 2014 (The Singapore Police Force, 29 Jan 2015), Available at http://www.police.gov.sg/img/stats/crimebrief2014.pdf?_ga=1.63579183.1069863767. 1440165618.

9. Couple lost son in Downtown East gang fight in 2010, but now saves his friends (The Straits Times, 23 Feb 2014), Available at http://www.straitstimes.com/singapore/couple-lost-son-in-downtown-east-gang-fight-in-2010-but-now-save-his-friends.

10. Youths attacked by '369' gang members (AsiaOne News, 9 Nov 2010), Available at http://news.asiaone.com/News/AsiaOne\%2BNews/Crime/Story/A1Story20101109246475.html.

11. Six youths arrested for Bukit Panjang attacks another 19 arrested for suspected involvement in gangs (The Singapore Police Force, 10 Nov 2010), Available at http:/ / www. police.gov.sg/mic/2010/11/20101110_bpanjang_attacker_arrested.html.

12. Youth stabbed after quarrel with friend (AsiaOne News, 5 Oct 2013), Available at http:// news.asiaone.com/news/crime/youth-stabbed-after-quarrel-friend.

13. 19-year-old youth stabbed outside Lot 1 shopping mall (STOMP, 2 Oct 2013), Available at http://singaporeseen.stomp.com.sg/singaporeseen/this-urban-jungle/youth-arrestedover-alleged-slashing-incident-at-lot-1-shopping-mall.

14. D. P. Farrington, Violence Vict. 4(2) (1989) 79-100.

15. D. P. Farrington, The Development and Treatment of Childhood Aggression, eds. K. H. Rubin and D. J. Pepler (Lawrence Erlbaum Associates, New Jersey, 1991).

16. T. E. Moffitt, Psychol. Rev. 100(4) (1993) 674-701.

17. T. N. Thornton, C. A. Craft, L. L. Dahlberg, B. S. Lynch and K. Baer, Best Practices of Youth Violence Prevention: A Sourcebook for Community Action (National Center for Injury Prevention and Control, 2000).

18. K. A. Dodge, Am. J. Prev. Med. 20(1) (2001) 63-70.

19. T. J. Taylor, J. Crime Justice 37(1) (2014) 1-4.

20. R. Loeber, D. P. Farrington and D. Petechuk, Child Delinquency: Early Intervention and Prevention (U.S. Department of Justice, Office of Justice Programs, Office of Juvenile Justice and Delinquency Prevention, 2003).

21. A. P. Goldstein, B. Glick, M. J. Irwin, C. Pask-McCartney and I. Rubama, Reducing Delinquency: Intervention in the Community (Pergamon Press, Oxford, 1989).

22. N. C. Klein, J. F. Alexander and B. V. Parsons, J. Consult. Clin. Psychol. 45(3) (1977) 469-474.

23. C. A. Smith and S. B. Stern, Soc. Serv. Rev. 71(3) (1997) 382-420.

24. A. J. Reynolds, S. R. Ou and J. W. Topitzes, Child Dev. 75(5) (2004) 1299-1328.

25. M. W. Lipsey, New Dir. Program Eval. 1988(37) (1988) 63-84.

26. M. D. Maltz, A. C. Gordon, D. McDowall and R. McCleary, Eval. Rev. 4(2) (1980) 225-240.

27. Y. Lee and T. S. Do, Int. J. Numer. Anal. Model. B 2(4) (2011) 369-378.

28. S. Peterson, The YVSP Strategy Lab. Youth Violence Systems Project, 2008, Available at http://www.egc.org/sites/egc.org/files/publications/err/issue_63_files/yvsp_sprev_ article2.pdf.

29. J. Sooknanan, B. Bhatt and D. M. G. Comissiong, J. Math. Res. 4(4) (2012) 10-27.

30. I. Munyo, Rev. Econ. Dyn. 18(2) (2015) 201-211.

31. M. W. Macy and R. Willer, Ann. Rev. Sociol. 28 (2002) 143-166.

32. E. Oh, K. Rho, H. Hong and B. Kahng, Phys. Rev. E 72(4) (2005) 047101.

33. K. Park, Y. C. Lai, S. Gupte and J. W. Kim, Chaos 16(1) (2006) 015105. 
34. A. Arenas, A. Díaz-Guilera and C. J. Pérez-Vicente, Phys. Rev. Lett. 96(11) (2006) 114102.

35. D. Li, I. Leyva, J. A. Almendral, I. Sendina-Nadal, J. M. Buldú, S. Havlin and S. Boccaletti, Phys. Rev. Lett. 101(16) (2008) 168701.

36. S. V. Buldyrev, R. Parshani, G. Paul, H. E. Stanley and S. Havlin, Nature 464 (2010) $1025-1028$.

37. Y. Hu, B. Ksherim, R. Cohen and S. Havlin, Phys. Rev. E 84 (2011) 066116.

38. M. M. Danziger, M. Shekhtman, A. Bashan, Y. Berezin and S. Havlin, Interconnected Networks, ed. A. Garas (Springer International Publishing, 2016).

39. L. M. Shekhtman, M. M. Danziger and S. Havlin, Chaos, Solitons Fractals 90 (2016) $28-36$.

40. A. Raine, K. Dodge, R. Loeber, L. Gatzke-Kopp, D. Lynam, C. Reynolds et al., Aggress. Behav. 32 (2006) 159-171.

41. K. G. Hill, J. C. Howell, J. D. Hawkins and S. R. Battin-Pearson, J. Res. Crime Delinq. 36 (1999) 300-322.

42. J. Wood and E. Alleyne, Aggress. Violent Behav. 15 (2010) 100-111.

43. B. B. Benda and C. L. Tollett, J. Crim. Justice 27 (1999) 111-126.

44. C. C. Cottle, R. J. Lee and K. Heilbrun, Crim. Justice Behav. 28 (2001) 367-394.

45. Y. Li and R. M. Lerner, Dev. Psychol. 47 (2011) 233-247.

46. S. Battin-Pearson, M. D. Newcomb, R. D. Abbott, K. G. Hill, R. F. Catalano and J. D. Hawkins, J. Educ. Pyschol. 92 (2000) 568-582.

47. R. P. Ang and V. S. Huan, Crim. Justice Behav. 35 (2008) 895-905.

48. R. P. Ang, E. Y. L. Ong, J. C. Y. Lim and E. W. Lim, Soc. Dev. 19 (2010) 721-734.

49. S. L. Seah and R. P. Ang, Aggress. Behav. 34 (2008) 553-562.

50. V. Dupéré, É. Lacourse, J. D. Willms, F. Vitaro and R. E. Tremblay, J. Abnorm. Child Psychol. 35 (2007) 1035-1045.

51. K. O'Brien, M. Daffern, C. M. Chu, and S. D. M. Thomas, Aggress. Violent Behav. 18 (2013) 417-425.

52. W. A. Mason and M. Windle, J. Abnorm. Psychol. 111 (2002) 63-76.

53. P. J. Hirschifield and J. Gasper, J. Youth Adolesc. 40 (2011) 3-22.

54. D. C. French and J. Conrad, J. Res. Adolesc. 11 (2001) 225-244.

55. T. P. Thornberry, A. J. Lizotte, M. D. Krohn, M. Farnworth and S. J. Jang, Criminology 32 (1994) 47-83.

56. E. E. Tanner-Smith, S. J. Wilson and M. W. Lipsey, The Oxford Handbook of Criminological Theory, eds. F. T. Cullen and P. Wilcox (Oxford University Press, New York, 2013).

57. P. J. Fite, C. R. Colder, J. E. Lochman and K. C. Wells, J. Clin. Child Adolesc. Psychol. 37 (2008) 412-421.

58. P. J. Fite, C. R. Colder, J. E. Lochman and K. C. Wells, Psychol. Addict. Behav. 21 (2007) 355-364.

59. R. P. Ang, V. S. Huan and D. Florell, J. Interpers. Violence 29 (2014) 237-254.

60. W. M. Craig, F. Vitaro, C. Gagno and R. E. Tremblay, Soc. Dev. 11 (2002) 53-68.

61. B. B. Benda, R. F. Corwyn and N. J. Toombs, Crim. Justice Behav. 28 (2001) 588-613.

62. R. P. Ang, V. S. Huan, S. H. Chua and S. H. Lim, Psychol. Crime Law 18 (2012) 703-711.

63. D. C. Pyrooz and S. H. Decker, J. Quant. Criminol. 29 (2013) 251-272.

64. J. C. Howell, Juvenile Justice Bulletin (Office of Juvenile Justice and Delinquency Prevention, Washington, 2010).

65. B. R. Maynard, C. P. Salas-Wright and M. G. Vaughn, Community Ment. Health J. 51 (2015) 289-299. 
66. K. L. Henry, K. E. Knight and T. P. Thornberry, J. Youth Adolesc. 41 (2012) 156-166.

67. J. N. Leaw, R. P. Ang, V. S. Huan, W. T. Chan and S. A. Cheong, PLoS ONE 10(6) (2015) 0126752.

68. R. P. Ang, V. S. Huan, W. T. Chan, S. A. Cheong and J. N. Leaw, J. Adolesc. 41 (2015) $148-156$. 